\title{
Potassium Iodide. The Solution to Silver Diamine Fluoride Discoloration?
}

\author{
Vinh Nguyen ${ }^{1}$, Cody Neill ${ }^{1}$, Joel Felsenfeld DDS ${ }^{2}$ and Carolyn Primus PhD ${ }^{3 *}$ \\ ${ }^{1}$ Third year students LECOM School of Dental Medicine in Bradenton, Florida \\ ${ }^{2}$ Assistant Professor of Restorative Dentistry LECOM School of Dental Medicine in Bradenton, Florida \\ ${ }^{3}$ Former Associate Professor of Dental Materials LECOM School of Dental Medicine
}

Submission: June 01, 2017; Published: June 15, 2017

*Corresponding author: Primus Consulting, Sarasota, FL, Email: Cprimus@me.com

\begin{abstract}
Introduction: Silver diamine fluoride (SDF) is increasingly recognized as a cost-effective, easy-to-use alternative to reduce sensitivity and arrest caries. However, SDF causes black staining of caries. Potassium iodide (KI) treatment with SDF may decrease or lessen the staining. However, the effectiveness of KI on staining has not been investigated. This study examined SDF/KI with various restorative materials, on carious and sound tooth structure.
\end{abstract}

Methods: Ten groups of teeth were treated to evaluate the effect of KI and SDF treatment. Visual examination and color measurements were used to evaluate color changes among the groups.

Results: All groups with KI treatment showed minimal to no staining after four weeks. Groups that underwent SDF treatment alone were noticeably darker $(\Delta \mathrm{L})$ compared with groups that had KI treatment. The staining varied among the groups of restorative materials; however, all of the teeth that received the KI treatment were lighter than teeth that only received the SDF treatment.

Conclusion: Treatment with SDF followed by KI had little to no darkening, compared to SDF treatment, when used with glass ionomer (self-cure), resin modified glass ionomer, composite, or no restorative on carious and sound teeth.

Practical implications: Silver diamine fluoride treatment followed by application of a saturated solution of potassium iodide markedly reduced staining compared to silver diamine fluoride alone, with or without common restorative materials.

Background: Adults' are poorly informed on how to care for children's teeth, according to a national survey conducted for the Children's Dental Health Project (CDHP) [1]. Key findings include that 2 out of 5 US adults believe they cannot prevent their child from developing caries, and few know when children should begin brushing or proper brushing techniques. Dental caries is the most common chronic childhood disease; five times more common than asthma, four times more common than childhood obesity, and twenty times more common than diabetes [2]. Forty two percent of US children between ages 2 and 11 have had dental caries in their primary teeth [3].

\section{Introduction}

Beyond our borders, early childhood caries is a global pandemic for children between ages of 6 and 12. For instance, in Mexico, $90 \%$ of children have caries; in the Philippines $92 \%$ of children are affected. Dentists treat dental caries with the "drill and fill" technique [4]. This surgical model requires the skill and equipment of a dentist in an operatory. Underserved areas where dentists and equipment are not available leave many children and adults without proper care for caries.

Until recently, no other treatment of dental caries has been available in the US besides preparation and restoration. Silver diamine fluoride (SDF) treatment offers an alternative caries paradigm. SDF is a colorless liquid agent with a $\mathrm{pH}$ of 10 containing $24.4 \%$ to $28.8 \%$ (weight/volume) silver and 5.0 to $5.9 \%$ fluoride [5]. SDF is indicated for desensitization; however,
SDF has off-label uses for reducing bacteria and arresting caries. Its anti-caries effect has been shown to be more effective than fluoride varnish [6], and can be used as a diagnostic indicator. Carious lesions treated with SDF increase in mineral density and hardness, and decrease in size [7]. SDF can be used in the demographics that suffer from the highest rates of tooth decay: children, bed-bound patients, people with mental disabilities, post-radiation and chemotherapy patients with xerostomia, and members of the community in very low socioeconomic situations. The low cost of SDF treatment and ease of the application make this a treatment option in dental offices and community dental health projects. Arresting active caries is a valuable service, which dental auxiliaries can be trained to perform. SDF can also be used in modern dentistry as a preventive treatment once or twice a year [8]. 
SDF is effective at arresting existing caries in the primary teeth of children, especially toddlers and children with behavioral issues [9]. In children, SDF applied annually is more effective at remineralizing teeth and preventing caries than fluoride varnish when applied every three months [10]. Similar results were seen in elderly patients, for caries prevention and arrest in root and occlusal surface caries, when applied annually [11]. Geriatric patients can be treated bedside using SDF for "drill-free bedside dentistry". While these techniques are not widely practiced in the US, SDF can be directly applied to tooth preparations before a restorative material is placed. Using SDF allows a more conservative tooth preparation to be made because the SDF has been shown to effectively arrest remaining decay, remineralize and harden leathery dentin [12].

Potassium iodide (KI) is widely used as a nutritional supplement. Supersaturated solutions of KI [13] have been tested as a second step after SDF treatment. The effectiveness of SDF in arresting caries was not affected, or minimally affected, by the application of KI. However, other research found that KI reduced the anti-caries effects of SDF by reducing the number of silver ions [14]. Caries arrest depends on the number of SDF applications to the affected lesion; reapplication of SDF after one year may be beneficial. Systematic reviews show that the best results for inhibiting caries is to reapply SDF to an affected area every 6 months for a period of 2 to 3 years [15].

The mechanism of how SDF arrests caries is not completely known; however, silver salts and silver ions are known to be antimicrobial [16]. Current research shows that silver ions inhibit the biofilm formation by inactivating and interfering with the bacterial synthesis of cellular polysaccharides. The silver interferes with the glycosyltransferase enzymes that synthesize glucan, which is the majority of the biofilm [17]. The glucan creates the adhesion of biofilm and resists microbial treatment [18]. The fluoride ions in SDF also contribute to its effectiveness. Fluoride is considered the gold standard for tooth remineralization since the 1980s. Fluoride inhibits acid demineralization by substituting in the enamel and dentin's apatite crystals, enhances remineralization, and inhibits the carbohydrate metabolism of acidogenic oral bacteria [19].

SDF is relatively new in the US but it has been used in other countries for over 80 years. One drop $(25 \mu \mathrm{L})$ of SDF contains $9.5 \mathrm{mg}$ silver diamine fluoride, enough to treat five teeth. Teeth should be treated no more than 3 weeks in a row and no more than every 6 months to meet the silver exposure guidelines set by the EPA [20]. No adverse incidents have been reported in Japan [21]. In an 18-month study in Hong Kong, 304 children with 1,670 tooth surfaces with dentin caries were treated with $\mathrm{SDF}$ or NaF varnish. None of the children who underwent the SDF treatment experienced adverse effects on the treated lesion or soft tissues [22]. In a review of nine randomized clinical trials with 1,493 children and elders, only 3 patients experienced "a small, mildly painful white lesion in the mucosa, which disappeared at 48 hours without treatment."
The major drawback to SDF treatment has been its black stain, which has limited its use. To counter the staining, researchers have tested SDF treatment immediately followed with a saturated solution of potassium iodide ( $10 \%$ weight $\% \mathrm{KI})$ followed by glass ionomer cement [23]. The bonding strength of glass ionomer (GI) restorative to dentin was not affected by the treatment with SDF/KI, if the precipitates were adequately rinsed away before application of the GI[13]. If the precipitate is not washed away, the bonding strength was significantly lowered. In another study, the pretreatment of dentine with SDF and KI minimized nanoleakage at the resin-dentin interface, assessed with a transmission electron microscope, without reducing the bond strength for either an etch and rinse or selfetch bonding system [24].

\section{Materials}

Advantage Arrest $^{\mathrm{TM}}$ SDF liquid (Elevate Oral Care, LLC, West Palm Beach, FL) was used which had been cleared by the FDA in 2014 for tooth desensitization. A saturated solution of potassium iodide (Upsher-Smith, Maple Grove, MN) known as Lugol's solution was purchased at an independent pharmacy. The KI solution is used medically as an expectorant, thyroid treatments, and in radiation emergencies. The KI solution was a colorless liquid agent containing $1 \mathrm{~g}$ KI per milliliter of water (10\% by weight). The restorative materials are described in the Methods.

Table 1: Treatment Groups.

\begin{tabular}{|c|c|c|c|c|}
\hline Group & Preparation & Restorative & SDF & KI \\
\hline A. & $\begin{array}{c}\text { Class I } \\
\text { Restoration }\end{array}$ & composite & $\mathrm{X}$ & $\mathrm{X}$ \\
\hline B. & $\begin{array}{c}\text { Class I } \\
\text { Restoration }\end{array}$ & composite & $\mathrm{X}$ & \\
\hline Control & $\begin{array}{c}\text { Class I } \\
\text { Restoration }\end{array}$ & composite & & \\
\hline C. & Existing Caries & none & $\mathrm{X}$ & $\mathrm{X}$ \\
\hline D. & Existing Caries & none & $\mathrm{X}$ & \\
\hline Control & Existing Caries & none & & \\
\hline E. & Caries-free & none & $\mathrm{X}$ & $\mathrm{X}$ \\
\hline F. & Caries-free & none & $\mathrm{X}$ & \\
\hline Control & Caries-free & none & & \\
\hline G. & $\begin{array}{c}\text { Class I } \\
\text { Restoration }\end{array}$ & RMGI & $\mathrm{X}$ & $\mathrm{X}$ \\
\hline H. & $\begin{array}{c}\text { Class I } \\
\text { Restoration }\end{array}$ & RMGI & $\mathrm{X}$ & \\
\hline Control & $\begin{array}{c}\text { Class I } \\
\text { restoration }\end{array}$ & RMGI & & \\
\hline I. & $\begin{array}{c}\text { Class I } \\
\text { Restoration }\end{array}$ & GI & $\mathrm{X}$ & $\mathrm{X}$ \\
\hline $\mathrm{J}$. & $\begin{array}{c}\text { Class I } \\
\text { Restoration }\end{array}$ & GI & $\mathrm{X}$ & \\
\hline Control & $\begin{array}{l}\text { Class I } \\
\text { restoration }\end{array}$ & GI & & \\
\hline
\end{tabular}




\section{Methods}

Forty-five extracted human molars were donated from private dental practices with no knowledge of personally identifiable information as per HIPAA standards. The teeth had been extracted as part of routine care outside of the institution. IRB exemption (protocol 24-152) was obtained through Lake Erie College of Osteopathic Medicine at Seton Hill. The teeth were immediately soaked in 3\% bleach solution 12 months prior to the start of the experiment. Before testing, the molars were removed from the bleach solution and washed with water to remove any solutes. The individual teeth were mounted in Whipmix ISO type 3 mounting stone (Whipmix, Louisville, KY) ensuring the entire crown was visible. Ten groups were assigned. Two groups $(\mathrm{N}=8)$ had existing caries while the remaining groups had minimal decay $(\mathrm{N}=32)$. One control tooth was prepared for each group using the same procedure without the use of SDF or KI $(\mathrm{N}=5)$. Table 1 has a summary of the ten groups.

Teeth in groups A and B were prepared for a class 1 composite restoration, $0.5 \mathrm{~mm}$ into dentin, with divergent occlusal walls and a stain-free dentinoenamel junction. A high-speed electric handpiece and a 330 carbide bur were used, followed with a slow-speed electric handpiece with a 56 carbide bur. Using a microbrush, SDF was applied to the preparation. Immediately following SDF application, KI was applied using a separate microbrush. On initial application of the KI, a white precipitate formed. The KI liquid was reapplied until no more white precipitation was observed to form. The teeth were washed with copious amounts of water. One-mm of high-viscosity, light-cured resin-modified glass ionomer (RMGI) (Riva Light Cure HV®, SDI, Australia) was applied over the pulpal floor surface and cured for 20 seconds using a Radii-cal LED curing light (Southern Dental Ind., Bayswater, Australia). A seventh-generation bonding system (Beautibond ${ }^{\circledR}$ Shofu, Kyoto, Japan) was applied over the RMGI surface and light-cured for 10 seconds. A nano-hybrid composite resin (Beautifil $^{\circledR}$, Shofu, Japan) was incrementally placed and cured for 20 seconds. Group B underwent the same class 1 preparation as group A, without the KI treatment and wash.

Groups C and D were comprised of teeth with occlusal caries. Using a microbrush, SDF was applied to the surface of the carious lesions for 1-2 minutes. For group C, KI was applied using a microbrush until no more white precipitate formed. The teeth were washed with copious amounts of water. Group D followed the same steps as group C without the use of KI. For Group E, SDF was applied on occlusal surface with no existing carious lesion for 1-2 minutes. Then the KI solution was applied with a microbrush, until no new white precipitate formed. The teeth were washed with copious amounts of water. Group F followed the same steps as Group E without the use of KI.
Group G followed the steps as group A, including treatment with SDF after the cavity preparation. Then the KI solution was applied with a micro brush, until no more white precipitate formed. The teeth were washed with copious amounts of water. The difference between groups A and G was that only a resinmodified glass ionomer was used as the restorative material. Group $\mathrm{H}$ underwent the same steps as group $\mathrm{G}$, without the use of KI. Group I followed the same steps of group A, including treatment with SDF after the cavity preparation. Then the KI solution was applied with a micro brush, until no more white precipitate was formed. The teeth were washed with copious amounts of water. The teeth were completely restored with a self-cure glass ionomer (Riva Self Cure GI, SDI, Australia). Group J underwent the same steps as group I without the use of KI.

All teeth were photographed immediately after treatment and weekly for four weeks. The Samsung UF- 130ST highresolution digital presenter camera was used to capture all of the images throughout the study. The digital presenter features a 1.3 mega pixel digital camera with $14 x$ optical zoom to provide images in 720p high definition.

Each tooth's color was measured using a Nix ${ }^{\mathrm{TM}}$ Pro color sensor (Hamilton, Ontario, Canada.). The color sensor blocks out ambient light and provides its own calibrated light source using industry standard $45 / 0^{\circ}$ measurement. The device provides color readouts in CIELAB and calculates color differences in $\Delta$ E2000. The Nix color sensor was used immediately following treatment and after four weeks. From the Nix color data, $\Delta \mathrm{L}$ and $\Delta \mathrm{E}$ values were recorded and compared among the groups.

\section{Results}

Forty teeth were treated with silver diamine fluoride, and one-half were also treated with KI. Figure 1 shows exemplary photos of the teeth from the 10 groups immediately after preparation, and 2 \& 4 weeks post-treatment. All teeth treated with only SDF darkened, characteristic of SDF treatments. The teeth treated with SDF and restored with composite and RMGI restoration (Group B) had significant staining immediately after light-curing. The materials changed from their initial A2 shade to a grayish color. No additional darkening was observed 4 weeks later. Group D with existing caries without the use of KI began to stain a few hours after application of SDF. Group F with no existing caries had staining in deep pits and grooves. Group H (having a cavity preparation, SDF and RMGI treatments), had visible discoloration. The teeth that had self-cured GI but no KI treatment (Group J) had visible staining in the marginal area of the restorations several hours after SDF application. All groups with KI treatment (Groups A, C, E, G, I) showed minimal to no staining over four weeks. The control teeth without SDF or KI treatment did not change over time. 


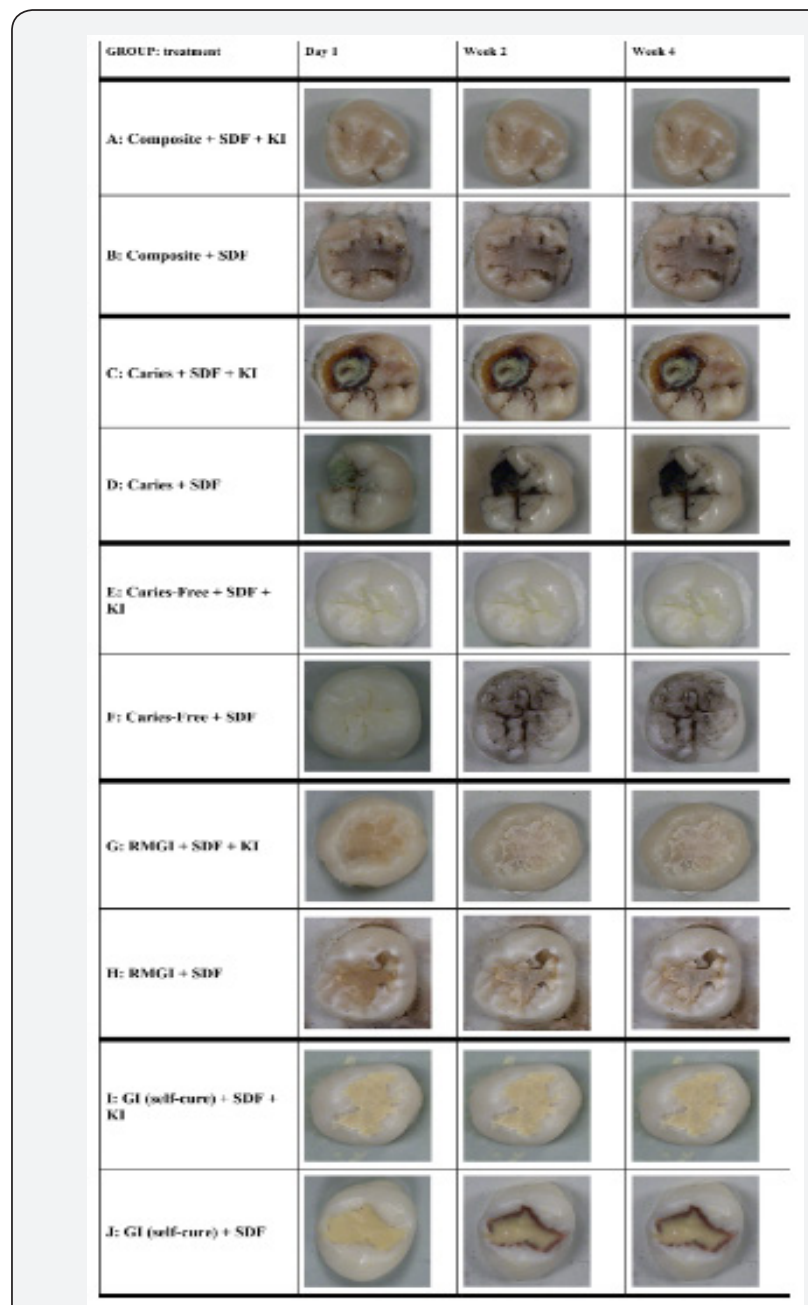

Figure 1: Exemplary photos of teeth from each treatment group.

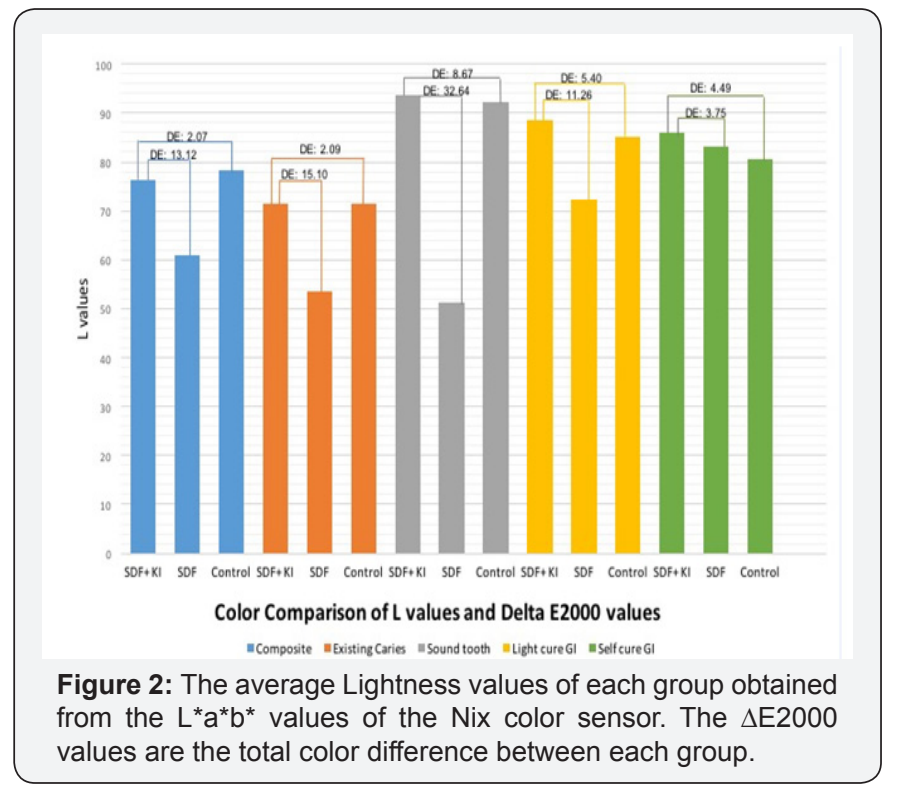

Table 2 has the values for lightness measured for each group. All teeth treated with only SDF showed darkening, reduction in $\mathrm{L}^{*}$, regardless of the restorative material used. The staining varied among the groups of restorative materials. All the teeth that received the KI treatment were lighter than teeth that only received the SDF treatment. Table 3 has the commonly accepted perceptible color differences. Figure 2 had the $\Delta \mathrm{L}$ and $\Delta \mathrm{E} 2000$ values for each group. The largest difference in the SDF and the SDF/KI treatments was observed for treatment of sound teeth. The smallest color differences were for the self-cured GI groups (I \& J). However, Group J had noticeable marginal staining. The teeth treated with SDF and KI were very similar in color to the control tooth for the group.

Table 2: Lightness Values.

\begin{tabular}{|c|c|c|c|c|c|c|c|c|c|c|}
\hline Group-> & A & B & C & D & E & F & G & H & I & J \\
\hline & 76.5 & 53.6 & 59.9 & 46.6 & 91.4 & 49.1 & 86.9 & 71.8 & 83.5 & 86.3 \\
\hline & 76.3 & 58.9 & 58.3 & 51.9 & 97.6 & 54.3 & 92.9 & 66.5 & 83.8 & 84.3 \\
\hline & 75.8 & 67.1 & 83.4 & 62.6 & 90.3 & 51.6 & 87.2 & 72.3 & 87.0 & 77.0 \\
\hline & 77.1 & 64.0 & 83.8 & 53.5 & 95.4 & 49.8 & 87.0 & 78.6 & 89.2 & 84.9 \\
\hline Average & 76.4 & 60.9 & 71.3 & 53.6 & 93.7 & 51.2 & 88.5 & 72.3 & 85.9 & 83.1 \\
\hline Std dev & 0.5 & 5.9 & 14.2 & 6.7 & 3.4 & 2.3 & 2.9 & 5.0 & 2.7 & 4.2 \\
\hline
\end{tabular}

Table 3: Commonly accepted color difference perceptibility

\begin{tabular}{|c|c|}
\hline$\Delta \mathbf{E}$ (CIE $\mathbf{L}^{*} \mathbf{a}^{*} \mathbf{b}^{*}$ units) & Visual Detection \\
\hline $0-1$ & No visible difference \\
\hline $1-2$ & Color difference not noticeable to untrained eye \\
\hline $3.5-5$ & Obvious shade difference when colors compared side by side \\
\hline $2-3.5$ & Color difference may be noticeable to an untrained eye \\
\hline$\leq 6$ & Cutoff value for commercially reproducible colors \\
\hline$>6$ & Different color \\
\hline
\end{tabular}


The application of the SDF material and the KI solution were simple to perform. However, upon application of SDF, an ammonia odor was noted. After the KI was added with the SDF material, the odor intensified. Air and water easily removed the white precipitates that occurred after KI treatment $[25,26]$.

\section{Discussion}

The chemical reaction of SDF has been proposed to be as in equation 1.

$$
\mathrm{Ag}\left(\mathrm{NH}_{3}\right) 2 \mathrm{~F}(\mathrm{aq}) \rightarrow \mathrm{Ag}(\mathrm{s})+2 \mathrm{NH}_{3}(\mathrm{~g})+\mathrm{F}^{-}(\mathrm{aq})
$$

The free fluoride ions are available to remineralize dentin and enamel; however, the silver precipitate is black. The chemical reaction of SDF treated with KI has been proposed to be as in equation 2 .

$$
\mathrm{Ag}\left(\mathrm{NH}_{3}\right) 2 \mathrm{~F}(\mathrm{aq})+\mathrm{KI}(\mathrm{aq}) \rightarrow \operatorname{AgI}(\mathrm{s})+2 \mathrm{NH}_{3}(\mathrm{~g})+\mathrm{F}^{-}(\mathrm{aq})
$$

Silver iodide is yellow, and insoluble in water. but the AgI precipitate is easily rinsed away. The SDF delivers the antimicrobial silver ions, but excess remain that can be precipitated as $\mathrm{Ag}_{2} \mathrm{~S}$ because the KI solution is applied after the $\mathrm{SDF}$, the excess silver ions are removed.

The curing light caused an immediate darkening of the SDFtreated areas, prompted by the photosensitivity of silver ions. However, the darkening did not worsen over the 4 weeks of the study. The photosensitivity of silver explains why the margins of the self-cured GI (Group J) developed darkening without light curing. From these results, we deduce that a curing light can be used to ensure that the KI treatment was successful, and ensures no darkening would occur. SDF was not supposed to stain sound tooth structure but in our experiment, the sound teeth were still stained by SDF treatment (Group F). This was attributed to using teeth soaked in bleach solution, causing a slight etch of the enamel.

Our color analyses support the use of KI treatment after SDF to eliminate the staining. However, continued studies with a larger sample size and a longer experimental period may be needed to confirm these results. In addition, studies including the placement of the test items in a salivary medium may give more detailed results as to what happens in the oral environment. At the end of the study, to ensure that no residual surface stain remained on sound tooth structure, the standard soft toothbrush (Colgate 360® Floss Tip Toothbrush, Colgate Oral Pharmaceuticals, New York, NY) and toothpaste (Colgate Total ${ }^{\circledR}$ Colgate Oral Pharmaceuticals, New York, NY) were used to brush the teeth; however, there were minimal changes on surface staining after brushing. Once the KI was added, a noticeable increase in ammonia odor was present presumably from the release of ammonia gas during the chemical reaction.

\section{Conclusion}

Treatments of teeth with KI solution after SDF treatment significantly reduced the discoloration caused by SDF. The effectiveness of this combination SDF/KI treatment should be clinically substantiated. If caries can be arrested or mitigated with this 2-step non-invasive treatment, a low-cost treatment may be more acceptable and practical for areas without full dental operatories, hospital patients, or for low-income pediatric patients with limited access to comprehensive dental care. The ammoniac odor created during SDF treatment should be disclosed to the patient.

\section{Acknowledgement}

We thank Dr. Mark Romer for providing the SDF material and Nicole Squitieri for procuring other essential materials.

\section{References}

1. https://www.cdhp.org/blog/392-survey-reveals-big-gaps-in-thepublic-s-knowledge-of-dental-health

2. Dye BA, Shenkin JD, Ogden CL, Marshall TA, Levy SM, Kanellis MJ (2004) The relationship between healthful eating practices and dental caries in children ages 2-5 years in the United States J Am Dent Assoc 135(1): 55-66.

3. Blue M, Christine (2012) Darby's comprehensive review of dental hygiene. ( $8^{\text {th }}$ edn) St. Louis: Elsevier, p. 631.

4. Bagramian RA, Garcia-Godoy F, Volpe AR (2009) The global increase in dental caries. A pending public health crisis. Am J Dent 22(1): 3-8.

5. http://www.ada.org/en/science-research/science-in-the-news/ silver-diamine-fluoride-in-caries-management

6. Lo EC, Chu CH, Lin HC (2001) A community-based caries control program for preschool children using topical fluorides: 18- month result. J Dent Res 80: 2071-2074.

7. Mei ML, Ito L, Cao Y, Li QL, Lo EC, et al. (2013) Inhibitory effect of silver diamine fluoride on dentine demineralization and collagen degradation. J Dent 41(9): 809-817.

8. Horst JA, Ellenikiotis H, Committee UCSFSCA, Milgrom PM (2016) UCSF Protocol for Caries Arrest Using Silver Diamine Fluoride: Rationale, Indications, and Consent. J Calif Dent Assoc 44(1): 16-28.

9. Chu CH, Lo EC (2008) Promoting Caries Arrest in Children with Silver Diamine Fluoride: A Review. Oral Health Prev Dent 6(4): 315-321.

10. Chu CH, Lo EC (2002) Effectiveness of Siler Diamine Fluoride and Sodium Fluoride Varnish in Arresting Dentin Caries in Chinese Preschool Children. J Dent Res 81(11): 767-770.

11. Yee R, Holmgren C, Mulder J, Lama D, Walker D, et al. (2009) Efficacy of Silver Diamine Fluoride for Arresting Caries Treatment. J Dent Res 88(7): 644-647.

12. Shiqian S, Zhang S, Mei ML, Lo E, Chu C (2016) Caries remineralisation and arresting effect in children by professionally applied fluoride treatment- a systematic review. BMC Oral Health 16: 12.

13. Knight GM, McIntyre JM, Mulyani (2006) The effect of silver fluoride and potassium iodide on the bond strength of auto cure glass ionomer cement to dentine. Aust Dent J 51(1): 42-45.

14.Zhao IS, Mei ML, Burrow MF, Lo EC, Chu CH (2017) Effect of Silver Diamine Fluoride and Potassium Iodide Treatment of Secondary Caries Prevention and Tooth Discolouration in Cervical Glass Ionomer Cement Restoration. Int J Mol Sci 18(2): E340.

15. Bowen D (2016) Effectiveness of Professionally-Applied Silver Diamine Fluoride in Arresting Dental Caries. J Dent Hyg 90(2): 75-78.

16. Rai M, Yadav A, Gade A (2009) Silver nanoparticles as a new generation of antimicrobials. Biotechnol Adv 27(1): 76-83. 
17.http://www.sciencedirect.com/science/article/pii / S0734975008000918

18. https://ann-clinmicrob.biomedcentral.com/articles/10.1186/14760711-12-4\#CR25

19. Nett J, Lincoln L, Marchillo K, Massey R, Holoyda K, et al. (2006) Putative Role of 1,3 Glucans in Candida albicans Biofilm Resistance. Antimicrob Agents Chemother 51(2): 510-520.

20. Nyvad B, Ten Cate JM, Fejerskov O (1997) Arrest of root surface caries in situ. J Dent Res 76: 1845-1853.

21. Vasquez E, Zegarra G, Chirinos E, Castillo JL, Taves DR, et al. (2012) Short term serum pharmacokinetics of diammine silver fluoride after oral application. BMC Oral Health 12(1): 60.

22. Horst J (2016) Silver diamine fluoride use in older adults. The journal of multidisciplinary care decisions in dentistry 2(8): 30.
23. Duangthip D, Chu CH, Lo CM (2015) A Randomized clinical trial on arresting dentine caries in preschool children by topical fluorides-18 month results. J Dent 44: 57-63.

24.Zhao IS, Mei ML, Burrow MF, Lo EC, Chu CH (2017) Effect of Silver Diamine Fluoride and Potassium Iodide Treatment of Secondary Caries Prevention and Tooth Discolouration in Cervical Glass Ionomer Cement Restoration. International Journal of Molecular Sciences 18(2): E340.

25. Selvaraj K, Sampath V, Sujatha V, Mahalaxmi S (2016) Evaluation of microshear bond strength and nanoleakage of etch-and-rinse and self-etch adhesives to dentin pretreated with silver diamine fluoride/ potassium iodide: An in vitro study. Indian J Dent Res 27(4): 421-425.

26. Paravina (2015) Color Difference Thresholds in Dentistry. Journal of Esthetic and Restorative Dentistry 27(S1): S1-S9.

\section{Your next submission with Juniper Publishers will reach you the below assets}

- Quality Editorial service

- Swift Peer Review

- Reprints availability

- E-prints Service

- Manuscript Podcast for convenient understanding

- Global attainment for your research

- Manuscript accessibility in different formats

( Pdf, E-pub, Full Text, Audio)

- Unceasing customer service

Track the below URL for one-step submission https://juniperpublishers.com/online-submission.php 